\title{
Atypical Presentation of a Giant Hemorrhagic Ovarian Cyst
}

\author{
Shashwati Sen ${ }^{1}\left[\right.$ Rajesh Maurya ${ }^{2} \cdot$ Gautam Sen $^{3} \cdot$ Harish Srivastava $^{4}$ \\ Received: 4 June 2021 / Accepted: 13 January 2022 / Published online: 17 February 2022 \\ (c) Federation of Obstetric \& Gynecological Societies of India 2022
}

\begin{abstract}
Hemorrhagic ovarian cysts (HOCs) are frequently seen in reproductive age women, developing in the luteal phase of ovulatory cycles. Giant HOCs are rare, as bleeding inside a closed space causes acute pain leading to early diagnosis. Anovulation or oligoovulation is a defining feature of polycystic ovarian syndrome (PCOS), and hence, functional cysts are uncommon in PCOS patients. This report describes a young girl with PCOS and amenorrhea, presenting with a giant hemorrhagic ovarian cyst. Most hemorrhagic cysts undergo spontaneous resolution with follow-up. Our patient presented as a surgical emergency following torsion and rupture with hemoperitoneum. Torsion causes an obstruction in the blood flow leading to infarction and loss of ovarian viability. Early diagnosis and detorsion may help to preserve ovarian function.
\end{abstract}

Keywords Hemorrhagic ovarian cyst · Corpus luteum cyst · Polycystic ovarian syndrome · Torsion · Hemoperitoneum · Whirlpool sign

\section{Introduction}

Hemorrhagic ovarian cysts (HOC) are most often luteal cysts resulting from bleeding into a corpus luteum. They are seen commonly in reproductive age women and develop in the luteal phase of ovulatory cycles. Clinical presentation varies widely and depends on the size of the cyst and associated complications. Smaller cysts are generally asymptomatic with spontaneous resolution within 6-8 weeks. Larger cysts are more painful because of increasing distention and are

Dr. Shashwati Sen is MBBS from Lady Hardinge Medical College, New Delhi and DGO from Maulana Azad Medical College, New Delhi. After completing her DNB (NBE), she pursued Postdoctoral Fellowship in Reproductive Medicine and Surgery at the prestigious Singapore General Hospital and Post Graduate Medical Institute, Singapore.

Shashwati Sen

Shashwati.sarkar@gmail.com

1 Department of Obstetrics and Gynecology, Mohak Hospital, Stanley Road, Muirabad, Prayagraj, U.P., India

2 Department of Anaesthesia, Mohak Hospital, Prayagraj, U.P., India

3 Department of Radiodiagnosis, Railway Hospital, Prayagraj, U.P., India

4 Department of Internal Medicine, Mohak Hospital, Prayagraj, U.P., India more prone to undergo rupture and torsion requiring immediate surgical intervention. Giant HOCs are rare, as bleeding inside a closed space causes acute pain leading to early diagnosis. Anovulation or oligoovulation is a defining feature of polycystic ovarian syndrome (PCOS), and hence, functional cysts are uncommon in PCOS patients. This report describes a patient with PCOS having prolonged and irregular cycles presenting with a giant hemorrhagic ovarian cyst. To our knowledge, this is the first of its kind to be described in the literature where a giant $\mathrm{HOC}$ has developed in a young girl with 2 months amenorrhea.

\section{History}

An 19 years old unmarried girl, presented to the emergency with sudden onset severe lower abdominal pain and vomiting for $4-5 \mathrm{~h}$, not relieved with analgesics. There were no associated bladder or bowel complaints. Her last menstrual period was 2 months ago, and she had history of prolonged and irregular cycles of 3-6 months over the past 2 years. She was diagnosed with PCOS about 1 year back but not on any treatment.

On examination, she was conscious and afebrile with a blood pressure of $90 / 60 \mathrm{mmHg}$ and respiratory rate of 20 breaths/min. Her body mass index (BMI) was 29, and there was the presence of coarse hair on her chin. On 
abdominal examination, there was rigidity in the right lower quadrant.

Laboratory investigations showed $\mathrm{Hb}-11.0 \mathrm{~g} / \mathrm{dl}$ and leukocytosis (TLC-14,000). Urine pregnancy test was negative. Ultrasound examination revealed an enlarged right ovary measuring $10.6 \times 8.2 \times 8.2 \mathrm{~cm}$ with predominantly diffuse solid pattern and areas of hypoechoic fluid pockets within it. Whirlpool sign suggestive of torsion of pedicle was noted on the ipsilateral side (Fig. 1). Left ovary had polycystic ovarian morphology. Uterus was normal in size and echo pattern with free fluid in pelvis.

Emergency laparotomy was performed in preference over laparoscopy due to concerns about COVID-19 virus transmission during laparoscopy from a potentially infected patient as recommended by all national and international endoscopic societies. A suprapubic transverse incision was given, and about $300 \mathrm{ml}$ of intraperitoneal blood and clots were removed. A $14 \times 10 \mathrm{~cm}$ hemorrhagic ovarian mass was noted on the right side of the uterus pushing the uterus to the left. The mass had ruptured along the inferior wall and was
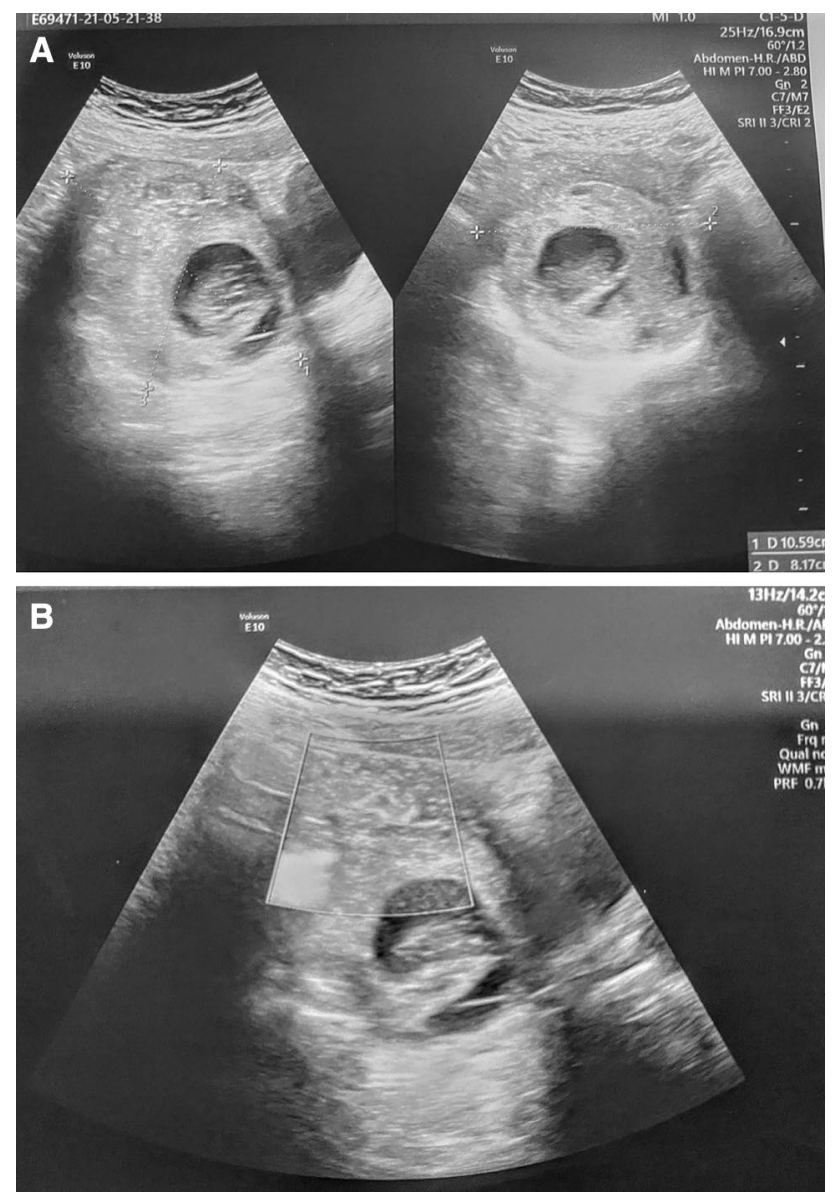

Fig. 1 a Scan showing hemorrhagic right ovary with hypoechoic fluid pocket within suggestive of necrosis. b Scan showing 'Whirlpool Sign' of torsion filled with blood clots. The right fallopian tube was edematous and dusky, lying posterior to the mass. Concomitant complete torsion of the tubo-ovarian mass along its supplying vascular pedicle had caused infarction and necrosis of the right fallopian tube and ovarian tissue (Fig. 2). The left ovary appeared enlarged and the left fallopian tube was healthy on inspection. Initial decision to salvage the ovary by detorsion was reviewed per operatively, and a right salpingooophorectomy was performed because of non-viability of the right adnexa. Postoperative period was uneventful and patient resumed her menses spontaneously on the 3rd postoperative day.

On histopathological examination, the cyst wall was lined partly by cuboidal cells and contained hemosiderin-laden macrophages and mononuclear cells. Features of congestion and necrosis were present. There were no endometrial glands/stroma and no malignancy.

\section{Discussion}

Formation of corpus luteum (CL) is a physiologic postovulation event in a regular cycling woman. It is a thinwalled vascular structure, and spontaneous bleeding within the cavity is usually self-limiting. Occasionally, excessive bleeding causes the CL to enlarge and form an hemorrhagic ovarian cyst.

It is unusual to have a giant $\mathrm{HOC}>10 \mathrm{~cm}$ as bleeding inside a closed space causes acute pain which forces the patient to seek medical help at the earliest. Rupture can lead to hemoperitoneum and presentation can be similar to a ruptured ectopic pregnancy. Giant cysts are also more likely to undergo torsion which can result in ischemic changes in the

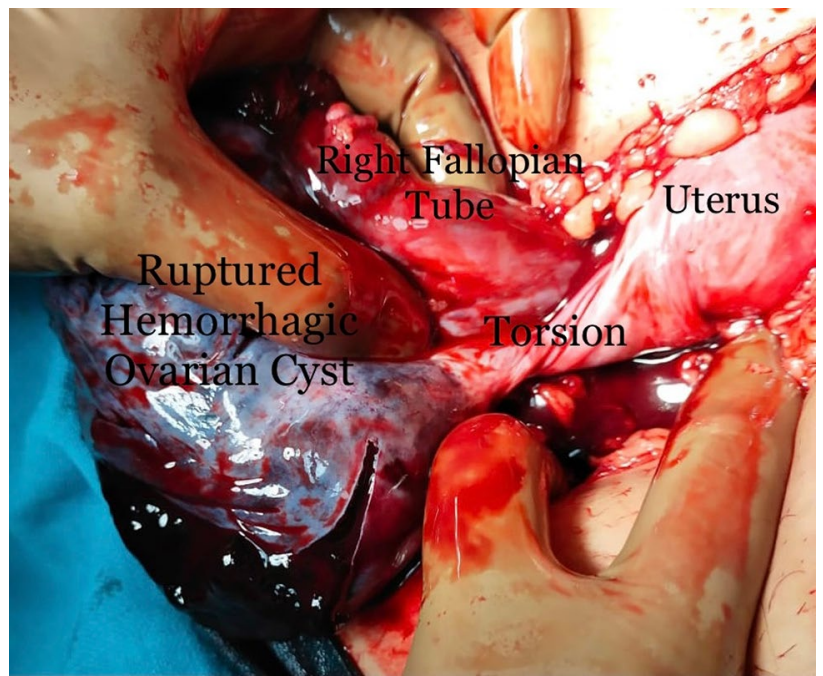

Fig. 2 Ruptured hemorrhagic ovarian cyst with torsion of pedicle 
ovary. Prolonged period of ischemia can lead to tissue death and loss of ovarian function.

Anovulation or oligoovulation is a feature of polycystic ovarian syndrome (PCOS), and hence, functional cysts are uncommon in PCOS patients. Our patient had an atypical presentation because she developed a giant hemorrhagic ovarian cyst in the presence of polycystic ovarian syndrome with prolonged and irregular cycles.

Durga et al. reported a giant hemorrhagic cyst $8.4 \times 5.3 \times 3 \mathrm{~cm}$ with torsion in a patient with regular cycles [1]. Abbas et al. in a retrospective review of HOCs reported a mean diameter of $4.8 \mathrm{~cm}$ with the largest being $8.7 \mathrm{~cm}$. $68.8 \%$ of the patients presented in the luteal phase and the remaining presented in the follicular phase [2]. There are other reports of hemorrhagic corpus luteal cyst which have all presented either in the luteal phase or follicular phase. It is rare to find ovarian mass in adolescent girls with PCOS and most are dermoid cysts. Our report is the first to our knowledge where an adolescent girl with PCOS presented with a giant hemorrhagic ovarian cyst during the amenorrhea phase.

The differential diagnosis to be considered is ectopic pregnancy, tubo-ovarian mass of PID, endometrioma and solid ovarian neoplasm. A urine pregnancy test is a must to rule out an ectopic pregnancy or a co-existent intrauterine pregnancy. Ultrasound is a critical first-line imaging modality for evaluating the size, volume and morphological pattern of the hemorrhagic cyst. The internal echogram can be classified into 3 patterns-

- Diffuse dense solid type

- Sponge-like pattern

- Mixed cystic-solid type

Hemoperitoneum appears as free hypoechoic fluid in the peritoneal cavity with focal hyperechoic collections suggesting blood clots. Color Doppler will show the absence of blood flow within the cyst with mild flow in the wall. A whirlpool sign is a definitive indicator of adnexal torsion and appears as a clockwise or counterclockwise enveloping of vessels around the central axis [3]. Although MRI is considered more sensitive for diagnosing pelvic pathologies due to high soft tissue contrast capability, yet low availability in an acute setting and high cost limit its utility.

Management depends on the size of the cyst and associated complications. Conservative approach with follow-up is adopted if the size is small. Larger cysts $(>5 \mathrm{~cm})$ which fail to resolve spontaneously or which present with acute symptoms of rupture, hemoperitoneum or torsion need surgical intervention. It is well established that ovarian cystectomy has a negative impact on the ovarian reserve with reduction in fertility potential. Anti-Mullerian hormone (AMH), a biochemical marker of ovarian reserve, can help to determine the postoperative fertility outcome. It is also important to understand that necrosis of ovarian tissue can itself cause a decline in AMH concentration. A preoperative AMH measurement can help in estimating the baseline levels and also in counseling. Our patient had prolonged torsion with necrosis of the tubo-ovarian tissue, and salpingo-oophorectomy was the best approach under such circumstances.

\section{Conclusion}

A twisted hemorrhagic ovarian cyst should be considered as a differential diagnosis after ectopic pregnancy has been ruled out, in a young PCOS girl presenting with acute pain abdomen and amenorrhea. Large luteal cysts with acute presentation are not known to develop in PCOS women and ours is the first report to the best of our knowledge. This is a surgical emergency, and a prompt and aggressive approach with adequate preoperative counseling is important in the successful management of the patient.

Funding The authors declare that no financial grants were received.

\section{Declarations}

Conflict of interest The authors declare that there is no conflict of interest.

Ethical approval This material is the authors' own original work and has not been previously published elsewhere. The paper is not currently being considered for publication elsewhere. The paper reflects the authors' own research and analysis in a truthful and complete manner.

Informed consent Informed consent was obtained from the patient for submission of the case report to the journal.

\section{References}

1. Durga K, Yasodha A, Yuvarajan S. Giant hemorrhagic ovarian cyst with torsion - rare case report. Obstet Gynecol Cases Rev. 2020;7:169.

2. Abbas AM, Amin MT, Tolba SM, et al. Hemorrhagic ovarian cysts: Clinical and sonographic correlation with the management options. Middle East Fertility Society Journal. 2016;21(1):41-5.

3. Singh D, Chinchure D, Chawla A. The "adnexal whirlpool" sign. Abdominal Radiology. 2016;41:2287-8.

Publisher's Note Springer Nature remains neutral with regard to jurisdictional claims in published maps and institutional affiliations. 


\section{About the Author}

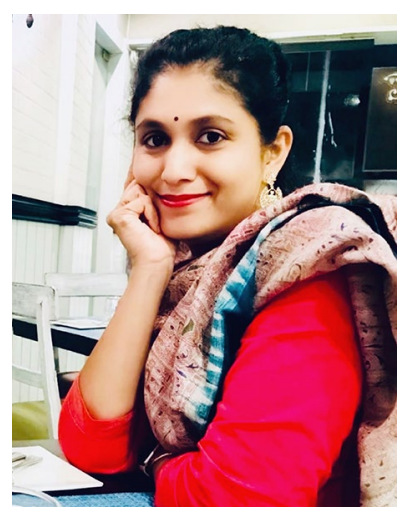

Dr. Shashwati Sen is MBBS from Lady Hardinge Medical College, New Delhi, and DGO from Maulana Azad Medical College, New Delhi. After completing her DNB (NBE), she pursued Postdoctoral Fellowship in Reproductive Medicine and Surgery at the prestigious Singapore General Hospital and Post Graduate Medical Institute, Singapore. She was a DNB guide with $\mathrm{KNMH}$, Prayagraj. She is on the Editorial Board of IJMA,
Al-Azhar University, Egypt. She is also a reviewer of eminence for many international journals. She has publications in Fertility and Sterility, Fertility and Reproduction (ASPIRE) and has presented papers and posters in ASPIRE and PCRS. She has participated as Faculty in AICOG and other national conferences. Presently she is with Mohak Hospital, Prayagraj. Her special interests are infertility and high risk pregnancy. 